\title{
Nuevas metodologías de innovación educativa mediante la relación entre Inteligencias múltiples, creatividad y lateralidad en educación infantil
}

\author{
Lorena Dólera Serrano, Fátima Llamas Salguero y Verónica López Fernández. Universidad \\ Internacional de la Rioja
}

\begin{abstract}
Recepción: 11 de septiembre de 2015 | Revisión: 12 de septiembre de 2015 | Aceptación/Publicación: 13 de octubre de 2015 Correspondencia: fatima.llamas@unir.net

Citar: Dólera Serrano, L., Llamas Salguero, F., y López Fernández, V. (2015). Nuevas metodologías de innovación educativa mediante la relación entre Inteligencias múltiples, creatividad y lateralidad en educación infantil. ReiDoCrea, 4, 311-328. [http://hdl.handle.net/10481/38450]

Resumen: En la actualidad, son muchos los métodos educativos implementados en la etapa educativa infantil que tratan de favorecer el desarrollo cognitivo en sus diferentes facetas (emocional, física, lógico-matemática, lingüística, musical, naturalista, espacial y social) desde una perspectiva creativa, motivadora y significativa del aprendizaje, exigiendo mayores competencias profesionales en nuestros educadores. El presente artículo parte de la necesidad de examinar en un grupo de alumnos/as de edades comprendidas entre 4 y 5 años, sus niveles de desarrollo en Inteligencias Múltiples, Creatividad y Lateralidad con la finalidad de extraer conclusiones, elaborar un programa de intervención, mejorar el sistema de enseñanza-aprendizaje y atender a la diversidad del alumnado. El análisis estadístico de los resultados, nos ofrece como datos más significativos niveles más bajos en Inteligencia Intrapersonal y Naturalista resultando muy acusada la deficiencia en esta última, más de un $88 \%$ de los alumnos presentan valores susceptibles de mejora en Creatividad con una lateralidad definida en un $90 \%$. Éste constituirá el punto de partida para la elaboración de proceso de intervención psicopedagógica encaminado a atender a las facetas cognitivas de los alumnos para poder estimular y potenciar aquellas que sean objeto de mejora.
\end{abstract}

Palabras clave: Creatividad | Lateralidad cerebral

New Methodologies of Educational Innovation through the Relationship between Multiple Intelligences, Creativity and Laterality in Early Childhood Education

Abstract: Today, many educational methods implemented in child education stage to try to promote cognitive development in its different facets (Emotional, Physical, Logical-Mathematical, Linguistics, Musical, Naturalist, Spatial and Social) from a creative perspective, motivating and meaningful learning demanding higher skills in our educators. This article assumes the need to examine a group of male / female students aged between 4 and 5 years, their levels of development in Multiple Intelligence, Creativity and Laterality in order to draw conclusions, develop an intervention program to improve the system of teaching and learning and to meet student diversity. In addition we intend to inquire into the relationship between Multiple Intelligence and Creativity and secondly between Creativity and settlement of laterality. The statistical analysis of the results, offers us as more significant information lower levels in Intelligence Intrapersonal and Naturalist turning out to be very marked the deficiency in the latter, more than $88 \%$ of the pupils presents values capable of improvement in Creativity with a laterally defined in $90 \%$. This one will constitute the point of item for the process production of intervention psycho-pedagogy directed to attending to the cognitive facets of the pupils to be able to stimulate and promote those that are an object of improvement.

Keywords: Creativity | Lateral Dominance

\section{Introducción}

En la actualidad, la etapa de Educación Infantil se configura como un periodo decisivo en la formación de la persona en sus diferentes facetas física, afectiva, social e intelectual en contraposición a la concepción tradicional a lo largo de la historia considerándola exclusivamente desde una perspectiva asistencial y caracterizada por una visión restringida de la educación.

De especial relevancia y como herramienta de reflexión resulta la publicación en 1996 del Informe a la UNESCO de la Comisión Internacional sobre educación para el siglo XXI presidida por Jacques Delors. De acuerdo con este informe, el volumen y la rapidez de los cambios económicos, culturales y científicos de nuestros tiempos hace que sea esencial la mejora de la formación de las personas y prepararlas para vivir en un mundo en constantes transformaciones, un mundo en el que la capacidad y las oportunidades para aprender a lo largo de la vida son fundamentales para el bienestar y desarrollo personal y social. De acuerdo con lo expuesto, resulta evidente el papel 
decisivo que desempeñan las competencias personales y profesionales los docentes en el marco educativo y en concreto la relacionada con la competencia científica. Esta requiere la consecución de una formación permanente del docente encaminada a conocer las últimas aportaciones, descubrimientos e investigaciones (atención a la diversidad, Inteligencias Múltiples, Proyectos Creativos, avances en Neuropsicología, etc.)

Por esta razón, nuestro motivo de estudio se centra fundamentalmente en partir del conocimiento y análisis de las Inteligencias Múltiples, Creatividad y Lateralidad en los alumnos/as y por medio de las conclusiones extraídas, elaborar un programa educativo acorde a sus características, necesidades y potencialidades educativas para mejorar la eficiencia del sistema de enseñanza-aprendizaje y aportar una óptica más innovadora y creativa. En relación a cada concepto procederemos a describir la documentación más relevante para contextualizar teóricamente la temática planteada en este trabajo.

La propuesta de Gardner (1983) psicólogo, investigador y profesor de la universidad de Harvard, a través de la teoría de las Inteligencias Múltiples destaca como una opción alternativa y candente dado su gran valor pedagógico en los últimos tiempos, y en concreto en la etapa educativa infantil. Howard Gardner muestra una visión pluralista de la mente y presenta ocho tipos de inteligencias en los individuos que trabajan de forma interrelacionada, incluso cuando una o más destacan, éstas son las siguientes:

- Inteligencia Lingüística. Se refiere a la adecuada construcción de las oraciones, la utilización de las palabras de acuerdo con sus significados y sonidos, al igual que la utilización del lenguaje de conformidad con sus diversos usos.

- Inteligencia Lógico-matemática. Referida a la facilidad para manejar cadenas de razonamiento e identificar patrones de funcionamiento en la resolución de problemas.

- Inteligencia viso-espacial. Referida a la habilidad para manejar los espacios, planos, mapas, y a la capacidad para visualizar objetos desde perspectivas diferentes.

- Inteligencia corporal-cinestésica. Señala la capacidad para manejar el cuerpo en la realización de movimientos en función del espacio físico y para manejar objetos con destreza.

- Inteligencia Musical. Se refiere al uso adecuado del ritmo, melodía y tono en la construcción y apreciación musical.

- Inteligencia Naturalista. Se refiere la habilidad para discriminar y clasificar los organismos vivos existentes en la naturaleza.

- Inteligencia Intrapersonal. Señala la capacidad que tiene una persona para conocer su mundo interno, es decir, sus propias y más íntimas emociones y sentimientos, así como sus propias fortalezas y debilidades.

- Inteligencia Interpersonal. Muestra la habilidad para reconocer las emociones y sentimientos derivados de las relaciones entre las personas y sus grupos.

La teoría de las Inteligencias Múltiples se organiza a la luz de los orígenes neurobiológicos de cada capacidad o habilidad para resolver problemas. Esta composición de inteligencias no resultan seleccionadas al azar ni por intuición sino que procede de diversos estudios que avalan la existencia de las mismas. En la siguiente tabla relacional se recoge la validación de los principios que sustentan la teoría de las Inteligencias Múltiples: 


\begin{tabular}{|l|l|}
\hline \multicolumn{2}{|c|}{ Tabla 1. Validación de los principios que sustentan la teoría de las Inteligencias Múltiples } \\
\hline PRINCIPIOS & FUNDAMENTACIÓN \\
\hline $\begin{array}{l}\text { Existencia de distintos estilos cognitivos autónomos e } \\
\text { independientes. }\end{array}$ & $\begin{array}{l}\text { Afectación de algunas inteligencias causadas por daño } \\
\text { cerebral relacionadas con las áreas específicas del cerebro } \\
\text { pertenecientes, quedando otras intactas. }\end{array}$ \\
\hline $\begin{array}{l}\text { Existencia de "sabios idiotas", prodigios y otros individuos } \\
\text { excepcionales. }\end{array}$ & $\begin{array}{l}\text { Muestran un claro perfil diferencial en cuanto a determinadas } \\
\text { inteligencias que destacan en un nivel alto frente a otras más } \\
\text { frágiles. }\end{array}$ \\
\hline $\begin{array}{l}\text { Cada inteligencia experta presenta su propia trayectoria } \\
\text { evolutiva. }\end{array}$ & $\begin{array}{l}\text { Se consideran aptitudes expertas (ej. Mozart como } \\
\text { compositor) y se analiza su inicio, desarrollo y declive. }\end{array}$ \\
\hline $\begin{array}{l}\text { Una historia evolutiva para cada tipo de inteligencia en en } \\
\text { función a las oportunidades de desarrollo. }\end{array}$ & $\begin{array}{l}\text { Por medio del estudio de las formas de inteligencia espacial } \\
\text { en mamíferos o inteligencia musical por medio de evidencias } \\
\text { arqueológicas en instrumentos musicales primitivos. }\end{array}$ \\
\hline Apoyo desde el campo de la psicometría. & $\begin{array}{l}\text { Las mediciones de los test psicométricos validan la existencia } \\
\text { aptitudes y capacidades diferenciadas. }\end{array}$ \\
\hline Respaldo de la psicología experimental. & $\begin{array}{l}\text { Investigaciones sugieren que las inteligencias son } \\
\text { independientes y poseen diferentes niveles de desarrollo. Ej. } \\
\text { Se puede tener una buena percepción de los sonidos } \\
\text { musicales y no de los verbales. }\end{array}$ \\
\hline La susceptibilidad de codificación. & $\begin{array}{l}\text { Cada inteligencia posee su propio sistema simbólico, tales } \\
\text { como el lenguaje, la pintura, las matemáticas, los mapas } \\
\text { como útiles de desempeño fundamentales. }\end{array}$ \\
\hline
\end{tabular}

Siguiendo en la línea de lo expuesto en la tabla anterior, Gardner (2001) añade que además cada inteligencia posee una operación nuclear identificable, o un conjunto de operaciones. Esto implica situándonos a modo de ejemplo, que en la inteligencia lingüística podemos extraer como operaciones identificables la sensibilidad ante los rasgos fonológicos, el lenguaje, lectura y escritura.

El motor que impulsó la formulación de la teoría de Gardner, no fue más que la falta de eficiencia y derrota de aquellos modelos psicológicos conductistas y psicométricos al servicio del campo de la educación. Estos modelos validan al sujeto desde una perspectiva pasiva, dependiente, cuantificable que desemboca en un modo de aprendizaje universal y determinado sin valorar previamente sus capacidades, potencialidades y sin ofrecer diferentes procedimientos de aprendizaje a la materia. Frente a esta postura, Gardner ofrece una alternativa diferente que parte del análisis de las capacidades más destacadas o más debilitadas del alumno/a para aprovechar este conocimiento y enseñar a los alumnos a gestionar sus inteligencias más desarrolladas para alcanzar una auténtica comprensión de lo aprendido y transferir este conocimiento. Aunque Gardner no diseñó ninguna teoría pedagógica al respecto, su interés y desarrollo por el desarrollo de las mentes infantiles viene reflejado en los proyectos pedagógicos de los que formó parte integrante (proyecto Zero 1967, proyecto MACOS 1960); su trabajo en la Escuela de Medicina de la Universidad de Boston; su compromiso con algunas teorías cognitivas de las que se nutre (Vigostky, Piaget, Bruner) y otras influencias de educadores relevantes (Montesori, Decroly). De todo este bagaje profesional podemos extraer sus aportaciones más relevantes a la Psicología de la Educación. Así pues, resultan especialmente interesantes y enriquecedores los programas y proyectos impulsados por la teoría de las Inteligencias Múltiples dirigidos a distintos colectivos y edades. Entre su variedad señalamos los siguientes: Proyectos de las Escuelas Keys, Proyecto Sumit, Programa de Inteligencia Práctica, Arts Propel, Aula Inteligente, The Fuller School y Proyecto Spectrum.

Por otro lado, nos situamos en el concepto de Creatividad que aunque se remonta desde la antigüedad es realmente en los años 50 cuando el término se consolida por Joy Paul Guilford (1897-1987). A partir de este momento se produjo un impulso al 
estudio de la creatividad desembocando en la aparición de diversas tendencias psicológicas (Cognitivas, Ambiental y motivacional, Histórico Cultural y Psicoanalistas).

Tratando de ofrecer un definición más completa del término de Creatividad, Rendón (2003) la define como "una capacidad y como todas las capacidades es un componente estructural de la personalidad que es susceptible de ser desarrollado, tiene bases neurológicas y características sociales. Los seres humanos comparten la capacidad creadora y las habilidades que la componen, no importa si son niños o adultos o si es en el campo de las artes, ciencia, la política o la industria. En este sentido, todos los individuos por naturaleza somos potencialmente creativos, y con la experiencia y el conocimiento llegamos a construir productos creativos que cumplan con criterios de novedad, a dar soluciones nuevas para nosotros mismos o para la sociedad y a salvar situaciones que se nos presentan".

Son diversos los autores que han tratado de recoger aquellas características que permitan una descripción más acertada del individuo creativo (Perkins, 1986; Ausubel, 1982; Mackinnon, 1980). A modo de síntesis podemos recopilar las siguientes características más destacadas en los individuos creativos: optimismo, pensamiento no convencional, motivación, libertad, originalidad, curiosidad, entusiasmo, autoconfianza, sentido crítico, iniciativa, persistencia, flexibilidad, imaginación, apertura, autocontrol, tolerancia a la ambigüedad, independencia.

Antes de continuar insistamos en los estudios neuropsicológicos acerca de la Creatividad, pues resulta un campo de investigación reciente y poco explorado. No obstante, se ha conseguido establecer algunas teorías interesantes. Son diversos los estudios que ponen de manifiesto una especialización hemisférica derecha en individuos creativos, otros evidencian que los individuos altamente creativos desempeñan un mayor flujo cerebral en ambos lóbulos frontales (Carlsson 2000) y en las investigaciones electroencefalografías se ha demostrado la incidencia de ondas de baja frecuencia alfa (periodos de relajación) y theta (corresponde a las primeras etapas de sueño) en personas creativas. A este respecto, conviene destacar la labor investigativa de Arieti (1976) y Rendón (2003) que definen tres estructuras cerebrales implicadas en la Creatividad como son la corteza temporo-occipito-parietal (TOP), la corteza prefrontal (CPR) y el sistema límbico.

La creatividad se encuentra estrechamente ligada al contexto y ambiente sociocultural y por tanto, puede ser estimulada en el entorno educativo. Amabile (1983) así lo señala concediendo un papel relevante a los factores sociales en la creatividad desarrollando un modelo compuesto por aspectos motivacionales, evaluación y recompensas.

Dada la importancia que nos aporta la aplicación práctica de la creatividad en las aulas conviene plantear cómo trabajar la creatividad y cuáles son las fases del proceso creativo. Wallas (1926) engloba el proceso creativo en cuatro estadios: preparación(toma de contacto y búsqueda de información al problema planteado); incubación(internalización inconsciente del problema);iluminación(representación del problema solucionado); y verificación(el sujeto asegura la validez y perfecciona su propuesta). Otra de las cuestiones más sugerentes en la actualidad es cómo sistematizar la creatividad en el aula. Resulta de gran utilidad la propuesta planteada por Sternberg y Lubart (1993) que exponen la confluencia de seis recursos para estimular la creatividad: inteligencia, conocimiento, personalidad, contexto, motivación y estilos de pensamiento. Es por ello, que merece la pena subrayar algunas experiencias educativas actuales de desarrollo creativo en las aulas destacando, entre 
otras, los proyectos de investigación, trabajos cooperativos, hipermedia, sistema experto, y modelo CAIT (Constructivo, Autorregulado, Interactivo, Tecnológico).

Siguiendo nuestra breve visión conceptual, nos adentramos en último lugar en el término de Lateralidad dada la relevancia que se le otorga como factor determinante en los aprendizajes escolares y dificultades en su desarrollo (disgrafía, disortografía, alteraciones lectoescritoras, etc.). No obstante el proceso de lateralización puede ser detectado para poder prevenir alteraciones y optimizar su evolución por medio de una adecuada intervención educativa.

Cabe señalar que el concepto de lateralidad hace referencia al predominio lateral y preferencia de uso de un lado del eje corporal frente al otro. Este dominio es debido a una adecuada comunicación y distribución de funciones entre ambos hemisferios cerebrales, siendo uno de ellos el que actúa como referente en determinadas funciones que promueven los aprendizajes. De esta forma el dominio funcional de una o otra parte simétrica del cuerpo puede observarse a nivel de manos, pies, ojos y oídos.

El desarrollo lateral en los individuos no surge al azar sino que posee una historia evolutiva propia atravesando las siguientes etapas (Ferré, Catalán, Casaprima y Mombiela, 2000):

- Etapa prelateral. Tiene presencia alrededor de los seis meses de edad. Implica una activación de ambos hemisferios cerebrales y se observa en la utilización de ambos lados del cuerpo en situaciones de tumbado, reptado y gateo.

- Etapa contralateral. Se trata de una fase que permite automatizar patrones de movimiento adecuados y voluntarios gracias a los avances que experimenta el Sistema Nervioso Central y el funcionamiento del Cuerpo Calloso. El niño ya es capaz de coordinar en sus desplazamientos el brazo derecho con la pierna izquierda y el brazo izquierdo con la pierna derecha. Esta fase se consolida a los tres años de edad.

- Desarrollo de la lateralidad. De los tres a los cinco años se activa la lateralidad y desde los cinco años a los diez es cuando se desarrolla. En esta etapa cada hemisferio cerebral se especializa en sus funciones y el Cuerpo Calloso activa la comunicación interhemisférica favoreciendo el aprendizaje.

En cuanto a la cuestión de qué tipos de lateralidad podemos encontrar en los individuos, son diversos los autores que atienden a distintas clasificaciones en función a la predominancia de una parte del cuerpo (diestro integral, zurdo integral, zurdería contrariada, ambidiextrismo, lateralidad cruzada); al segmento corporal (lateralidad ocular, lateralidad manual, lateralidad pedal, lateralidad expresiva); a los gestos (de utilización o predominancia manual en acciones cotidianas, espontánea); y por último atendiendo a su naturaleza (normal o patológica).

\section{Método}

Una vez tratados los aspectos más destacados en torno a los que gira este trabajo de investigación, es preciso retomar que esta labor está dirigida al campo profesional de la educación y a la acción educativa partiendo de un análisis de las Inteligencias Múltiples, Creatividad y Lateralidad en alumnos del tercer nivel de educación infantil para ofrecer en función a los resultados, un sistema de enseñanza-aprendizaje acorde a las características, necesidades y potencialidades de los niños desde una visión innovadora, crítica y creativa de la enseñanza. 
Asimismo considerando el objeto principal de estudio que nos acontece procederemos a mencionar los siguientes objetivos específicos encaminados a tal fin:

- Detectar a través del conocimiento de las Inteligencias Múltiples los puntos fuertes y débiles del alumnado.

- Valorar el nivel de creatividad en el alumnado.

- Ajustar el método educativo a las necesidades, potencialidades y demandas de los alumnos a través de los resultados obtenidos.

- Elaborar un proyecto de intervención creativo para mejorar los niveles de Inteligencias Múltiples, Creatividad y Lateralidad en el tercer nivel de Educación Infantil.

El presente trabajo de estudio selecciona como muestra de referencia al alumnado de educación infantil y en concreto de manera más intencional se ha seleccionado a 30 sujetos del segundo ciclo de educación infantil en edades comprendidas entre los 4 y 5 años de edad de los que 11 son niñas y 19 niños. Nuestro contexto se sitúa en una CEIP de titularidad pública inserto en una zona urbana cuyo nivel socio-cultural se caracteriza por niveles medios-altos.

El diseño propuesto pretende incidir en el análisis de hechos ya sucedidos a través de la observación e interpretación de datos obtenidos enfocados a la acción y mejora educativa. Por consiguiente, esta investigación se nutre de una metodología no experimental, correlacional, cuantitativo-cualitativa y descriptiva.

En lo que se refiere a las variables objeto de análisis y los diferentes instrumentos utilizados para medir dichas variables en esta investigación se muestran las siguientes:

- Inteligencias Múltiples. Cuestionario del profesor para diagnosticar las Inteligencias Múltiples (Armstrong, 1999. Adaptación de Prieto y Ballester, 2003) (véase anexo 1) El cuestionario trata de averiguar los niveles en cada una de las ocho Inteligencias múltiples de los alumnos por medio de diez ítems expresados en términos de conductas. Estos son evaluados por la tutora en torno a su presencia o ausencia en el comportamiento de los alumnos/as. La puntuación corresponderá a 1 punto si identifica al alumno con este comportamiento, de 0,5 si identifica al alumno/a en algunas ocasiones y 0 si está conducta no está presente en el alumno/a. La suma total de todas las puntuaciones nos permitirá valorar el nivel del alumno/a en cada una de las Inteligencias Múltiples.

- Creatividad. Cuestionario de Creatividad (Dade Country Public Schools South Central District, Miami, Florida, Tuttle, 1980) (véase anexo 2). Es un cuestionario administrado para profesores compuesto por 7 items expresados en términos de conductas observables en los niños/as apuntándolas en las siguientes escalas de valoración: raramente, de vez en cuando, frecuentemente y casi siempre.

- Lateralidad. Test a nivel individual de lateralidad de la prueba neuropsicológica (adaptado por Martín Lobo, Castellón, Rodríguez y Vallejo, del equipo del Instituto de Neuropsicología y Educación, Fomento). Se emplea a partir de los 4 años. Esta prueba permite anotar la parte simétrica del corporal que domina en la visión, audición, mano y pie. 


\section{Resultados y discusión}

Tras aplicar los instrumentos de medida de las variables neuropsicológicas procederemos a citar los resultados obtenidos en las pruebas, con esta información se elaborará un análisis estadístico de los mismos para más adelante proceder a la fase de reflexión e interpretación.

De esta manera en Inteligencias Múltiples se mide el nivel de cada inteligencia por alumno/a del grupo-aula.

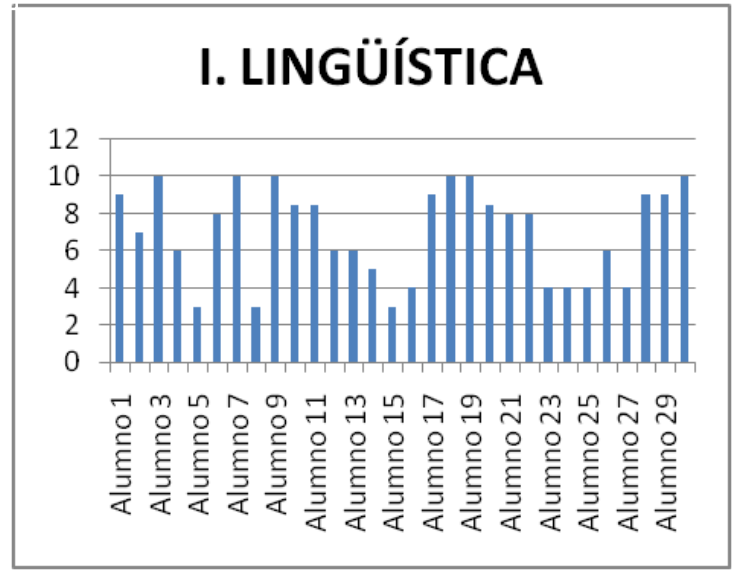

Figura 1. Inteligencia Lingüística

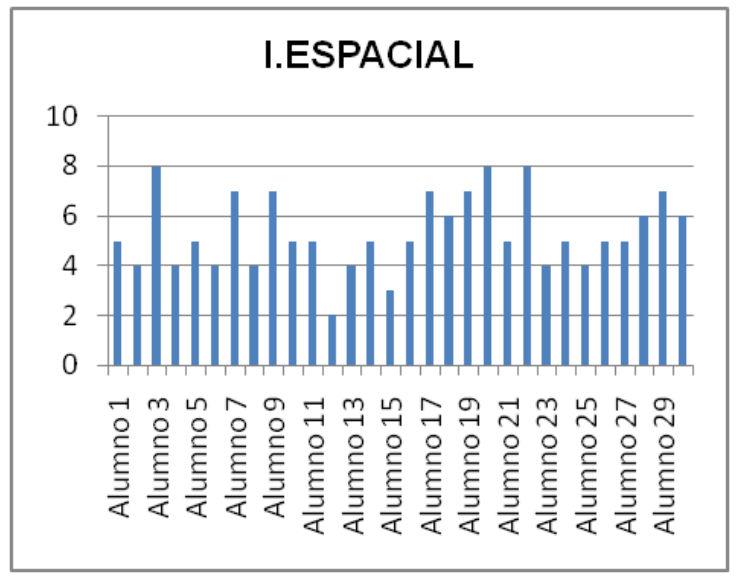

Figura 3. Inteligencia Espacial

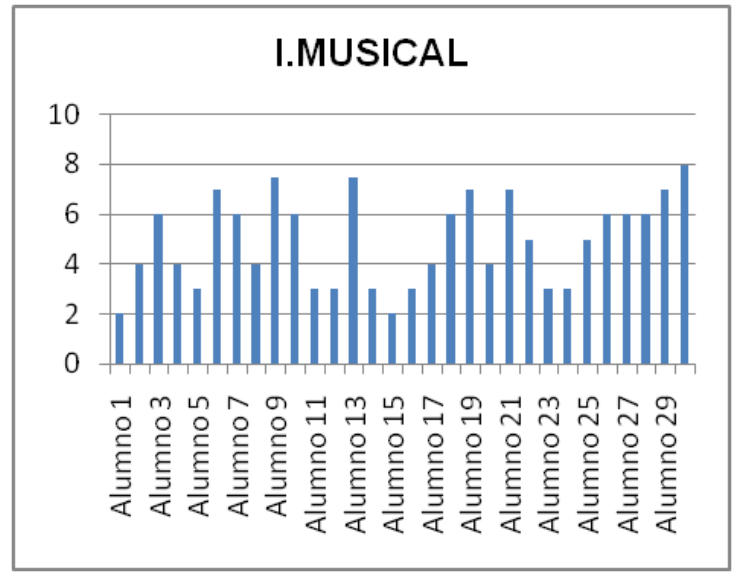

Figura 5. Inteligencia Musical

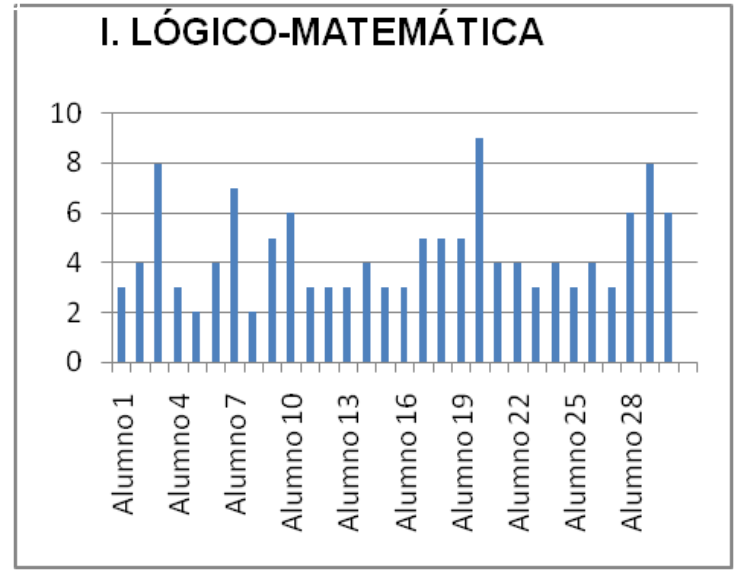

Figura 2. Inteligencia Lógico-matemática

I. CORPORAL-KINESTÉSICA.

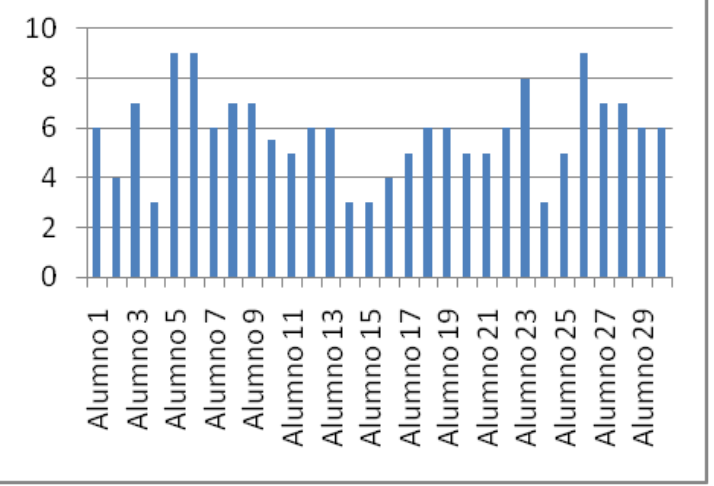

Figura 4. Inteligencia Corporal-kinestésica

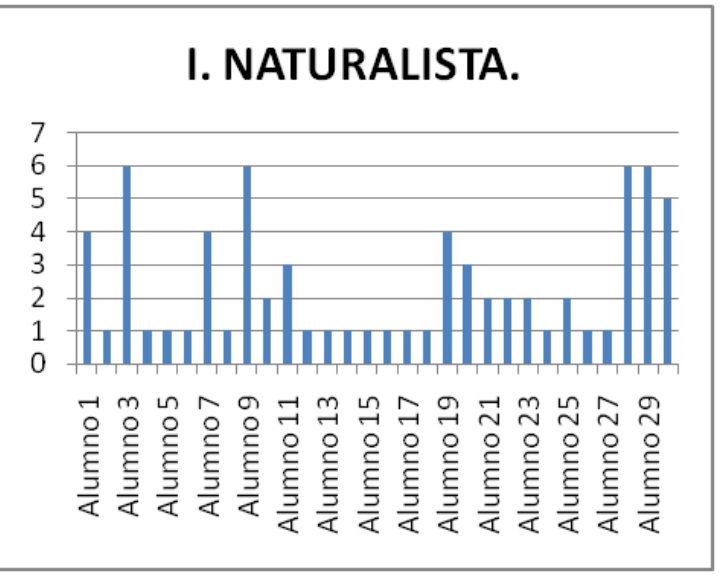

Figura 6. Inteligencia Naturalista 


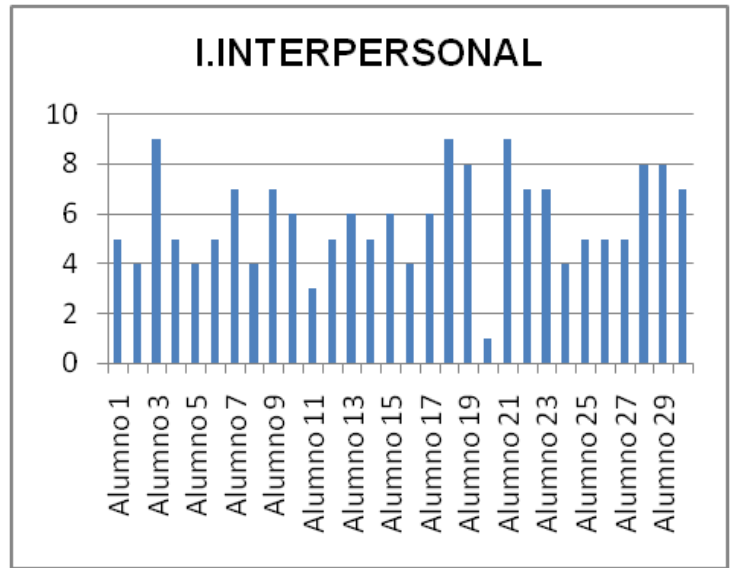

Figura 7. Inteligencia Interpersonal

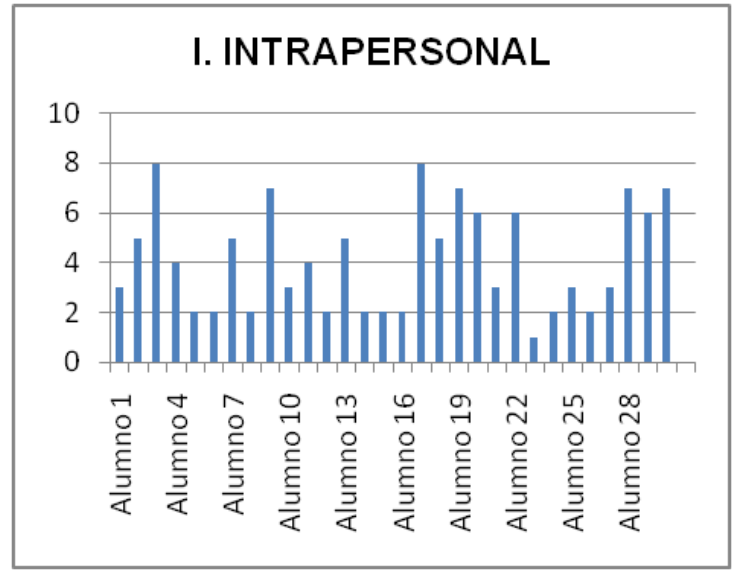

Figura 8. Inteligencia Intrapersonal

Proseguimos con el siguiente gráfico del promedio de puntuación del alumnado en cada una de las Inteligencias Múltiples.

\section{PROMEDIO DE LA MUESTRA EN INTELIGENCIAS} MÚLTIPLES

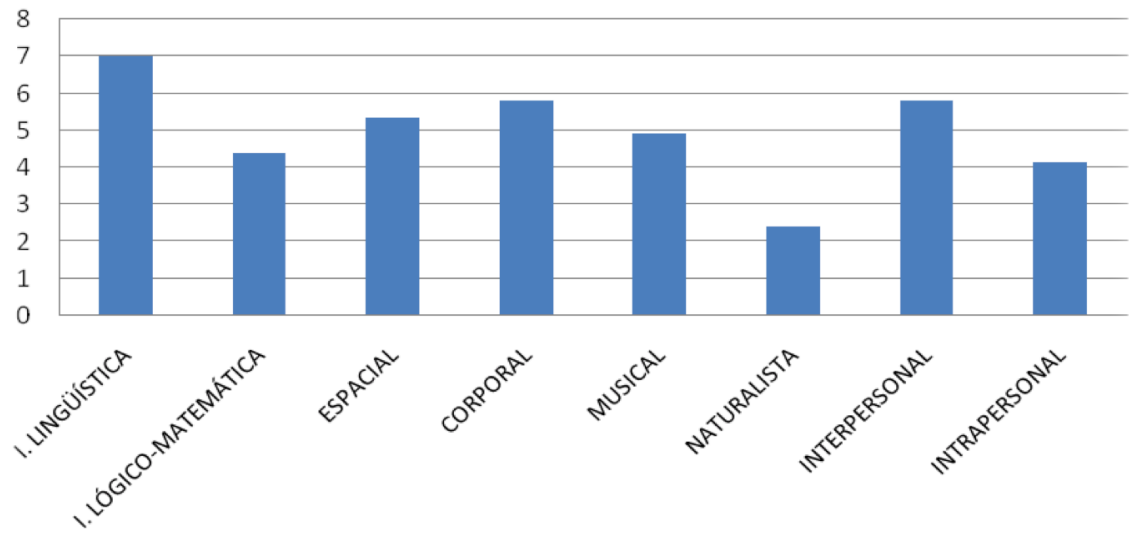

Figura 9. Gráfico de puntuación media en Inteligencias Múltiples

Los resultados muestra niveles de valoración por la tutora medio-altos en la mayoría de las inteligencias salvo en la naturalista que presenta las puntuaciones más bajas y objeto de mejora educativa. Pues así queda expuesto en la siguiente tabla 2:

\begin{tabular}{|lccccc|}
\hline \multicolumn{7}{c|}{ Tabla 2. Análisis estadístico en Inteligencias Múltiples } \\
\hline & I.LINGÜÍSTICA & I.LÓGICO-MATEMÁTICA & ESPACIAL & CORPORAL & MUSICAL \\
\cline { 2 - 7 } Promedio & 7,016 & 4,4 & 5,333 & 5,816 & 4,933 \\
Mediana & 8 & 4 & 5 & 6 & 5 \\
Moda & 10 & 3 & 5 & 6 & 6 \\
Desviación típica & 2,496 & 1,811 & 1,516 & 1,704 & 1,813 \\
\hline
\end{tabular}

Por otro lado, se procede a realizar un análisis estadístico tras los resultados obtenidos en los cuestionarios de creatividad. A continuación presentamos unos gráficos de frecuencias y porcentajes del grupo de alumnos/as en función a los valores más señalados por la tutora. 


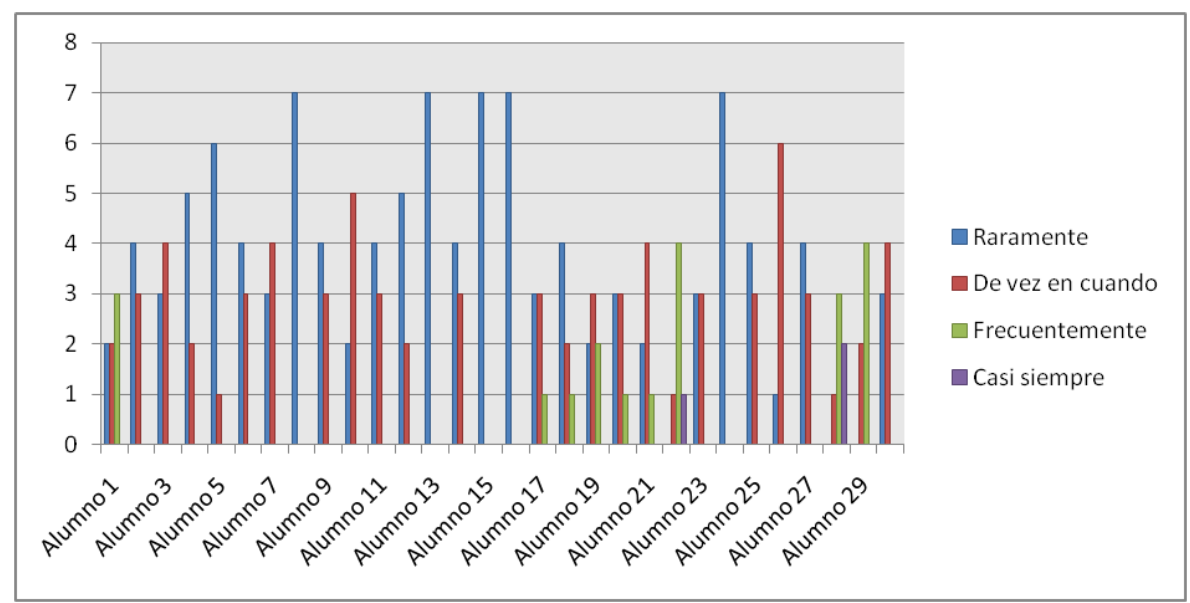

Figura 10. Gráfico de frecuencias en Creatividad de la muestra

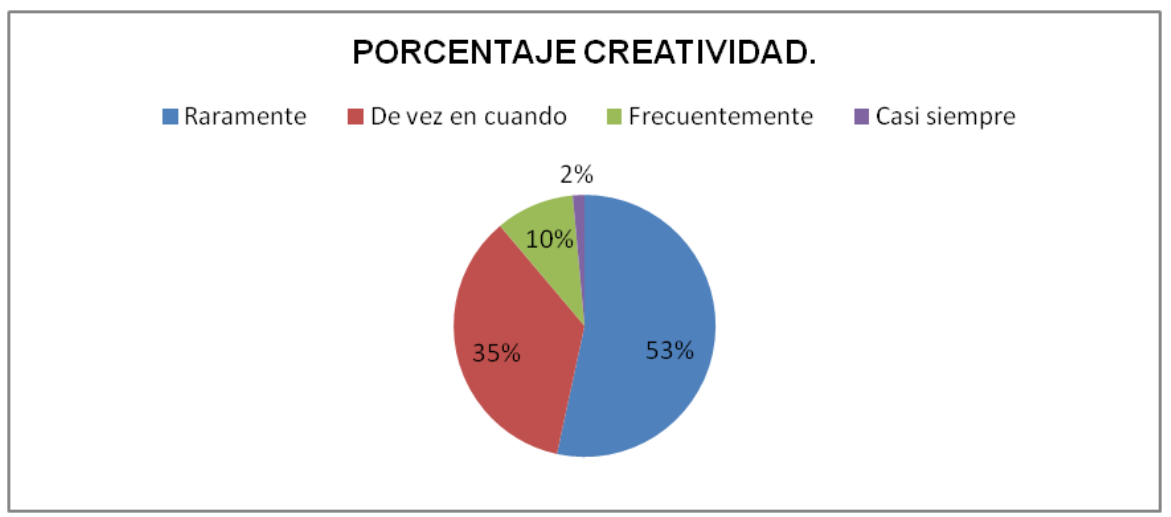

Figura 11. Porcentaje de la muestra en Creatividad

Este gráfico pone de manifiesto que un $53 \%$ del alumnado presenta raramente signos de creatividad, un $35 \%$ de vez en cuando, un $10 \%$ frecuentemente y un $2 \%$ casi siempre.

Por último, centramos el análisis de funciones estadísticas en lo que respecta al desarrollo lateral en los alumnos que componen nuestra muestra seleccionada. Los resultados obtenidos evidencian que un $90 \%$ del alumnado presenta un desarrollo lateral definido.

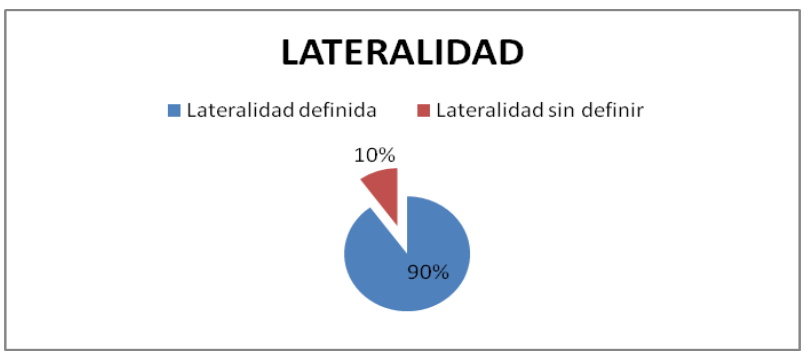

Figura 12. Porcentaje de Lateralidad en el alumnado

\begin{tabular}{|lccc|}
\hline \multicolumn{4}{c|}{ Tabla 3. Funciones estadísticas en Lateralidad } \\
\hline LATERALIDAD & Frecuencia & Porcentaje válido & Porcentaje acumulado \\
\hline Diestro & 26 & 86,666 & 86,666 \\
Zurdo & 1 & 3,333 & 90 \\
Diestro con cruce visual & 3 & 10 & 100 \\
\hline
\end{tabular}


Por todo ello se propone el siguiente programa de intervención con el fin de mejorar el proceso educativo. Conviene retomar aquellos aspectos que en este caso serán objeto de estimulación y mejora, pues nos situamos tras analizar los resultados obtenidos con deficiencias en el proceso de aprendizaje de la Inteligencia Naturalista de manera más acusada que requerirá mayor protagonismo en nuestro proceso de intervención, no obstante conviene potenciar esta inteligencia a través de aquellas que se presentan como puntos fuertes en el alumnado, además de los aspectos relacionados con la lateralidad con el objeto de estimulación y seguir en beneficio del adecuado desarrollo de las mismas aportando algunas pequeñas actividades complementarias de carácter anual.

Trataremos de mantener una continuidad e inclusión de este proceso de actuación con la programación educativa que la tutora ha establecido para el curso escolar evitando rupturas metodológicas innecesarias.

El programa de intervención neuropsicológica parte de los siguientes objetivos fundamentales a favorecer y conseguir en el alumnado:

- Atender a los diferentes estilos de aprendizaje y motivación de los alumnos/as y compensar aquellos aprendizajes relacionados con la Inteligencia Naturalista.

- Acercar a los alumnos/as al mundo científico por medio del método científico: observación, planteamiento de hipótesis, experimentación, resultados y conclusiones.

- Observar y explorar de forma activa su entorno y los fenómenos que lo rodean, extrayendo interpretaciones sobre situaciones $y$ hechos significativos $y$ mostrando interés por su conocimiento.

Nuestra opción metodológica consiste en adentrar en el conocimiento del mundo científico a los alumnos por medio de una renovación del rincón de experiencias que además incluirá nuevos materiales y pasará a transformarse en el rincón científico.

Todos los experimentos serán explicados por la tutora en la asamblea y en gran grupo, motivándolos, ayudándolos a realizar sus anticipaciones e hipótesis, permitiéndoles la observación y participación directa y comprobación de resultados y conclusiones. Tras esta toma de contacto, los alumnos/as desempeñarán estos ejercicios de manera autónoma, en pequeño grupo en el rincón científico y siempre bajo la supervisión de la tutora y finalizarán la tarea a través de la representación gráfica de lo experimentado.

La elección de esta propuesta metodológica se concreta en los siguientes principios que formularé a continuación:

- Aprendizaje significativo y materiales. A través de la observación de lo que sucede en su entorno y de la manipulación y experimentación de los materiales que están a su alcance, el niño/a irá desarrollando su sentido de la curiosidad y de la observación, lo que le ayudará a comprender mejor los fenómenos naturales, físicos y técnicos.

- La actividad infantil como fuente de aprendizaje. La secuencia de propuestas vivenciales debe posibilitar establecer semejanzas y diferencias, identificar, reconocer cambios, comprender fases y procesos, utilizaciones..., y, sobre todo, efectuar valoraciones de la realidad.

- La importancia del juego dentro de cada actividad.

- La interacción entre iguales.

- Atención a la diversidad del alumnado. 
En cuanto a las actividades propuestas vamos a distinguir aquellas destinadas al rincón científico y aquellas complementarias que tratarán de compensar la Inteligencia Naturalista por medio de las inteligencias más fuertes y destacadas y favorecer a su vez el desarrollo de la lateralidad.

\section{Sesión 1. ¿Quiénes son los científicos?}

En la zona de asamblea, hablaremos con los alumnos/as acerca de lo que saben sobre los científicos, quiénes son, a qué se dedican, cuáles son sus utensilios de trabajo, cómo visten, si conocen algún científico, etc. Tras esta recogida de las primeras impresiones y conocimientos previos de los alumnos/as presentaremos un visionado proyectado en la Pizarra Digital Interactiva sobre algunos científicos destacados, sus aportaciones, lugar de trabajo y utensilios. Además los haremos partícipes de la nueva experiencia en la que nos vamos a adentrar "la creación en el aula del rincón científico". Aprovecharemos para enviar una nota informativa y de colaboración a los padres y familiares acerca del nuevo rincón científico para aportar materiales (de reciclaje, batas, guantes, gafas, recipientes, imanes...) y búsqueda de información sobre la ciencia para decorar el rincón.

\section{Sesión 2. Salida a un laboratorio}

Esta actividad consistirá en la salida a un laboratorio de un instituto cercano al centro. El profesor de ciencias nos explicará qué es el laboratorio, para qué sirve, qué se puede hacer en él, de qué materiales se compone; y manejaremos por grupos diferentes útiles (microscopios, lupas, balanzas, guantes, batas, gafas).

\section{Sesión 3. Cromatografía con rotuladores}

El proceso químico por el que somos capaces de separar las diferentes sustancias químicas que componen una mezcla recibe el nombre de cromatografía. Esta actividad consiste en hacer una cromatografía de la tinta de rotulador para descomponerla en los diferentes pigmentos que contiene. Para llevar a cabo esta actividad necesitamos de los siguientes materiales: papel absorbente de cocina, filtros de café, rotuladores, vasos de plástico un poco de agua y pinzas de la ropa. Comenzamos recortando en forma de círculo los filtros del café cada uno de los niños, después pintaremos en el centro una figura (círculos, cuadrados, triángulos, rectángulos...) conviene usar el mismo color cada alumno para poder comparar mejor los resultados entre ellos. Tras ello, cogemos un vaso de plástico y le colocamos un papel absorbente al que empapamos bien con agua, ahora doblamos los círculos de papel por la mitad y los enrollamos formando un cono. Colocamos la punta del cucurucho dentro del vaso en contacto con el papel absorbente y sujeto a la pared del vaso con una pinza. Veremos finalmente como el dibujo de tinta original se ha descompuesto en los colores que lo componen. Además comprobaremos que los colores primarios no se descomponen (azul, rojo y amarillo) y otros como el lila, marrón, naranja, verde sí que se descomponen.

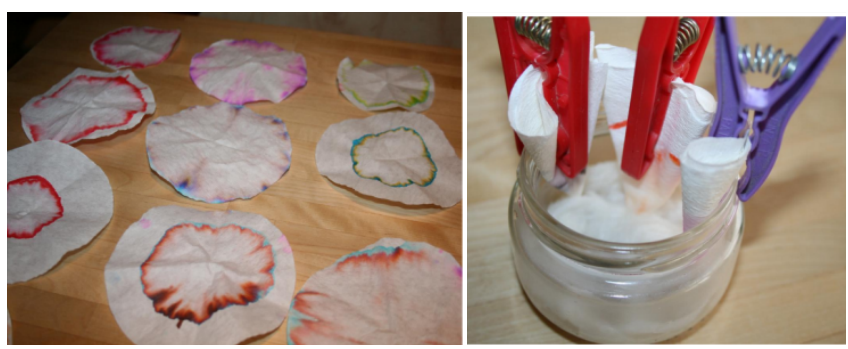

Figura 13. Cromatografía 


\section{Sesión 4 y 5 . Electricidad estática}

Los materiales previstos para estos experimentos son los siguientes: jerséis de lana, latas de refrescos vacíos, globos y sal. Para la puesta en práctica de estos experimentos ha sido necesario en primer lugar, cargar un globo electrostáticamente y frotándolo con insistencia sobre un jersey de lana. Lo que ocurre es que algunos electrones del jersey se traspasan al globo y quedan atrapados en su superficie cargándose negativamente. A continuación y una vez cargado el globo procederemos a las siguientes tareas en torno a ello con el resto de materiales tal y como puede verse en las imágenes.
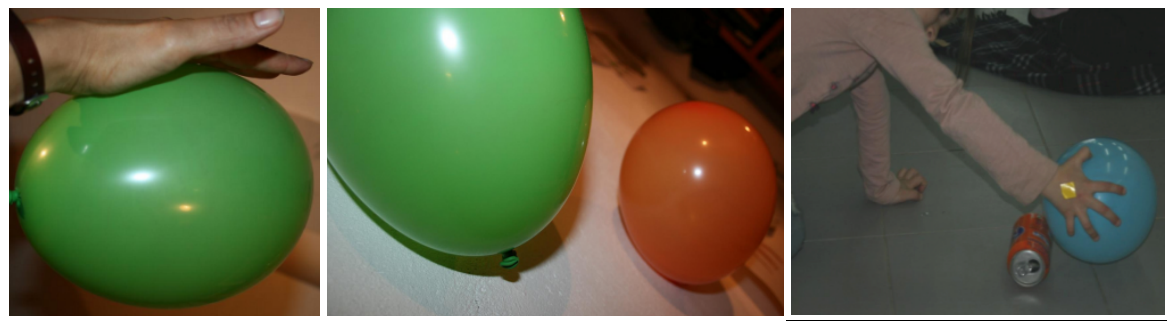

Figura 14. Electricidad estática

\section{Sesión 6 y 7. Juegos con imanes}

Como actividad inicial en la asamblea la tutora se colocará un imán en el bolsillo y por la parte exterior atraerá algunos objetos de forma mágica tras ello, aprovecharemos para enseñarles el pequeño truco y explicarles brevemente la fuerza del magnetismo. A continuación llevaremos a cabo varias actividades:

- Experimentación con objetos hechos con diferentes materiales.

- Esta vez vamos a comprobar el alcance de la fuerza de magnetismo de un imán.

- Y por último trataremos de comprobar cómo actúa la fuerza magnética cuando interponemos un material entre el imán y el objeto atraído.
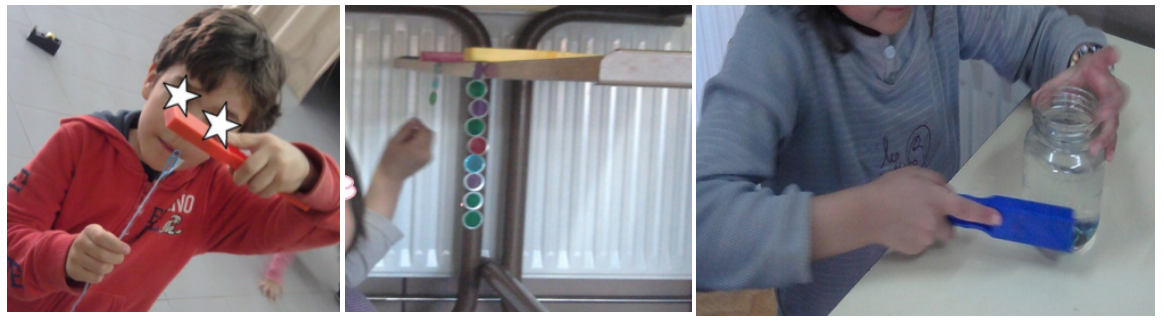

Figura 15. Juegos con imanes

\section{Sesión 8 y 9. Tensión superficial}

Esta actividad sobre la tensión superficial es una variante del experimento con leche, colorantes y jabón, pues en lugar de leche emplearemos cola.

Este experimento de tensión superficial en los líquidos es una propiedad por la que las moléculas de la superficie interaccionan con las moléculas que tienen por debajo, de manera que se forma una especie de película o lámina sobre la que pueden flotar objetos ligeros. En este caso, el jabón tiene la propiedad de rebajar la tensión superficial, es decir, hace que las fuerzas sobre la superficie sean menores en el punto de contacto. 

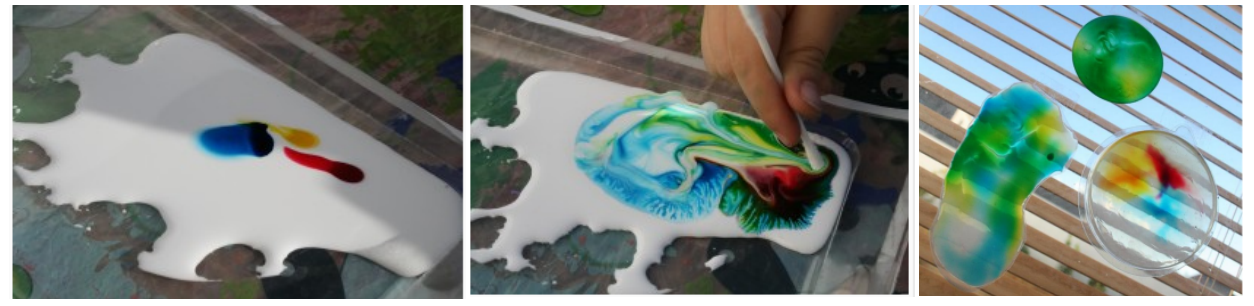

Figura 16. Tensión superficial

\section{Sesión 10. El pincha globos mágico}

Esta actividad consistirá en atravesar por un globo un pincho de madera sin hacerlo explotar. Para ello emplearemos los siguientes materiales: globos, pincho de madera largo para atravesar el globo, y un poco de aceite.

Este ejercicio requiere de mucha concentración, pues comenzaremos inflando el globo y anudándolo, ahora untamos con aceite la punta del pincho para suavizarlo y pinchamos el globo por la zona más cercana al nudo de manera suave y girando a ambos lados hasta atravesar el globo. Continuaremos hasta llegar al extremo opuesto al nudo hasta que la punta atraviese fuera del globo.

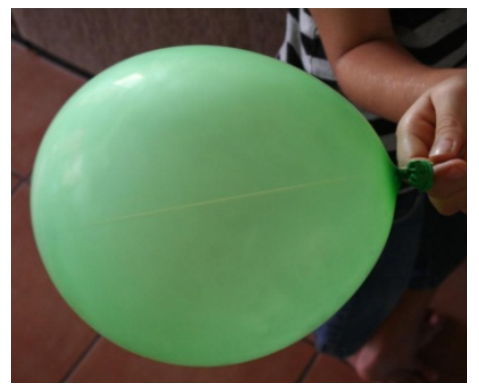

Figura 17. El pincha globos mágico

\section{Sesión 11. Huevos gelatinosos}

Este experimento será muy sencillo, vamos a necesitar dos huevos más o menos del mismo tamaño, dos tarros de cristal para introducir los huevos, vinagre y agua.

Colocamos un huevo en cada uno de los recipientes, ahora los vamos a cubrir uno con agua y el otro con vinagre en el rincón científico. Intentaremos explicar que puede pasar con cada uno de ellos y de esta manera extraer algunas hipótesis de nuestros alumnos/as.

Si dejamos pasar 3 días podremos observar qué ocurre, el huevo sumergido en vinagre produce burbujas alrededor de la cáscara que va haciendo que desaparezca la misma, entonces comprobaremos que ha cambiado su aspecto si lo extraemos y limpiamos con un paño con cuidado, ipues ha perdido la cáscara y ha aumentado de tamaño! ¡Parece un huevo de goma! Ahora nos preguntamos qué habrá ocurrido dentro de él, para ello, enfocaremos al huevo con una linterna y veremos que la yema sigue flotando en su interior.

El siguiente paso es extraer con cuidado el huevo sumergido en agua, y podremos ver que sigue intacto. Trataremos de comparar ambos huevos.

De esta forma comprobaremos que el huevo gelatinoso puede botar en una superficie lisa a corta altura mientras que el otro romperá tal y como ya sabemos. 
La explicación del huevo gelatinoso viene dado que se ha producido una reacción química entre las sustancias del vinagre y de la cáscara que producen dióxodo de carbono ( las burbujas) y al no tener cáscara entra el líquido en el huevo que le hace crecer por un proceso que se llama difusión.

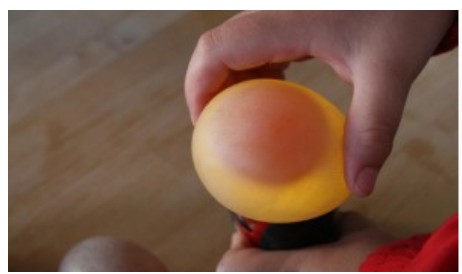

Figura 18. Huevos gelatinosos

\section{Sesión 12. El arcoíris de las plantas}

Previo al experimento hemos tratado de explicarles a los niños algunos conceptos importantes sobre el ciclo vital de las plantas, y de entre todos los contenidos recordaremos que las plantas absorben el agua y que esta se distribuye por el tallo hasta las hojas. Pues bien este experimento será muy representativo de esta explicación dada.

Necesitaremos 5 tarros altos para introducir los tallos de apio, colorante alimentario, y agua.

Comenzaremos llenando de agua a la mitad de su capacidad los tarros y en cada uno de ellos le introduciremos un colorante. Después le colocaremos los tallos de apio y dejaremos esperar un día, veremos cómo el apio no solo ha absorbido el agua sino que también se ha teñido del color del colorante.
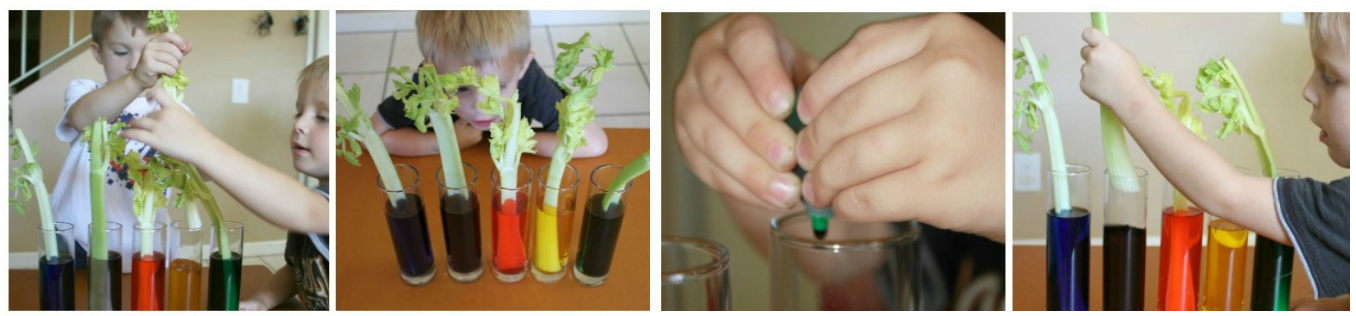

Figura 19. El arcoíris de las plantas

\section{Sesión 13. Salida a un vivero de plantas}

Asistimos a la visita de un vivero y conoceremos los diferentes tipos de plantas (hasta plantas carnívoras), fases de crecimiento, necesidades vitales, cuidados, útiles para cuidarlas y escucharemos los consejos de los profesores jardineros/as. Esta sesión nos servirá de enlace para la siguiente actividad pues nos regalarán unas orugas en un bote de cristal con una buena ración de comida (col) encontradas en una col del pequeño huerto del que disponen para poder experimentar y conocer más sobre ellas.

\section{Sesión 14. La oruga de la col}

Con las orugas en el tarro de cristal exponemos a los alumnos/as que este ser vivo necesita de alimentos (col) de oxígeno (agujeritos en la tapa del bote de cristal) y de un lugar fresco dentro del aula. Hablamos acerca de su ciclo vital, qué pasará al cabo de los días surgiendo la hipótesis de que se convertirá en una mariposa y para ello debemos dejar pasar los días y aportar entre todos su alimento y registrar todo lo 
acontecido en el tiempo. La actividad finalizará con el cuento de:" la pequeña oruga glotona".

\section{Sesión 15. Experimentos con el agua $1^{a}$ parte. Descubrimiento sensorial}

Salimos con los alumnos/as al patio del colegio y tenemos preparada una piscina de plástico llena de agua. Realizaremos las siguientes actividades:

- Tocaremos el agua con nuestras manos

- Jugaremos con diferentes recipientes

Todos estos ejercicios irán acompañados de reflexiones sobre el agua en cuanto al color, forma, sensaciones que producen a los sentidos.

Finalmente incluiremos una pequeña bañera de agua junto con los recipientes para que los alumnos/as sigan experimentando en el rincón científico.

\section{Sesión 16. Experimentos con el agua $2^{a}$ parte. ¿Flota o se hunde?}

En clase y haciendo uso de la pequeña bañera de agua del rincón científico, colocaremos a su lado una bolsa de objetos variados que iremos describiendo (esponja, bayeta de cocina, cucharas de plástico, madera y objetos de metal). A continuación, trataremos de averiguar por medio de hipótesis la siguiente premisa: ¿estos objetos flotan o no flotan en la bañera de agua? Lo comprobaremos y registraremos los resultados en el rincón científico.

\section{Sesión 17 y 18. Experimentos con el agua $3^{a}$ parte. Disoluciones}

En este experimento jugaremos a mezclar el agua con distintos materiales previamente mostrados por la tutora. Estos pueden ser entre otros: agua, café, detergente, leche, ajo, pintura, colorantes alimenticios, etc. Para ello contaremos con varios recipientes con tapa llenos de agua. Sólo le indicaremos las siguientes condiciones tendrán que meter dentro de los recipientes sólo un material, lo cerraremos y una vez bien cerrado agitamos y observamos lo que pasa. Tendremos que discernir aquellos materiales que se disuelven de los que no.

\section{Sesión 19. Experimentos con el agua $4^{a}$ parte. Del hielo al agua}

La actividad comienza presentando a los alumnos/as una bolsa llena de cubitos de hielo, algunos en su interior llevan pequeños objetos (bolitas, botones, muñequitos...) hablamos sobre lo que ellos conocen del hielo y sus vivencias, es decir, para qué lo usan, si les gusta o no, en qué casos es muy útil el hielo, etc. A continuación, dejamos que manipulen los hielos y describimos sensaciones, tras ello, les preguntaremos qué pasará si echamos los cubitos a la bañera de agua del rincón, ¿flotará o no? Mientras hablamos sobre ellos pondremos a prueba la observación de los alumnos/as y comprobaremos si alguno observa cambios en los hielos dentro del agua y de qué forma pueden salir los pequeños objetos dentro de los cubitos. Dejamos pasar el tiempo y comprobamos que solo quedan los pequeños objetos y no hay ni rastro del hielo. La siguiente cuestión girará en torno a cómo podemos volver a convertir el agua en hielo, para ello necesitamos cubiteras que llenaremos a la mitad de agua señalándolo con un rotulador para después introducir en el congelador. También veremos cómo crece en volumen el hielo con respecto a lo marcado en la cubitera con el agua. 


\section{Sesión 20. Experimentos con el agua $5^{a}$ parte. El agua de la lluvia}

Para poder explicar cómo ha llegado el agua de la lluvia a las nubes puede resultar útil el siguiente experimento. Necesitamos una bolsa de plástico con cierre hermético, la llenamos de agua y pegamos la bolsa a una ventana con cinta adhesiva y a partir de este momento solo se trata de observar al cabo de unas horas. Veremos cómo aparecen pequeñas gotitas en las paredes interiores de la bolsa como si se tratasen de gotas de lluvia. ¿Qué ha ocurrido? Intentamos explicar el proceso. Ahora explicamos a los niños que la temperatura del interior de la bolsa al efecto del sol en la ventana ha aumentado y el agua ha comenzado a evaporarse porque no puede escapar de la bolsa, al igual que ocurre en la atmósfera.

\section{Sesión 21. Salida al museo de la ciencia y el agua de la Región de Murcia}

En el presente museo realizaremos una visita guiada complementada con variadas actividades para el alumnado. Dispone de página web para más información: http://www.cienciayagua.org/.

Una vez finalizada la propuesta de intervención dedicada a la Inteligencia Naturalista a través del rincón de trabajo, dispondremos de algunas actividades complementarias dedicadas a compensar la Inteligencia Naturalista por medio de tres de las inteligencias que se presentan como puntos más fuertes para el grupo que citamos a continuación:

- Actividad de inteligencia emocional: el mural animal de los sentimientos. Presentamos a los alumnos un mural que viene representado en una tabla con varias columnas en las que aparecen imágenes de animales mostrando diferentes emociones (miedo, aburrimiento, cansancio, tristeza, vergüenza, enfado, sorpresa, nerviosismo, alegría y enfado) y todas sus fotos a un lado. Cada día de la semana en la asamblea expresaremos nuestros sentimientos, explicaremos los motivos y trataremos de describir con precisión cada estado de ánimo, tras esta reflexión tendremos que imitar al animal que coincida con ese estado y colocar su foto en la columna asignada a esa emoción.

- Actividad de inteligencia lingüística: álbumes de las estaciones. Elaboramos álbumes de las estaciones del año. Para esta actividad hemos de dedicar al menos una salida al patio del colegio por cada estación. Cada uno con una bolsita recogerá un poco de tierra, hojas, hierbas, flores... según encuentre en cada estación. Cuando llegamos a clase hacemos una puesta en común incidiendo en las características propias de los recursos obtenidos en la recolecta, hablamos de sus características: humedad, temperatura, color, textura asociándolo a la influencia de los cambios estacionales en el paisaje. Tras ello, pegaremos las hojas, tierra y demás recursos en folios A3 y las plastificaremos de esta forma elaboraremos la portada del álbum del otoño, primavera, verano e invierno.

- Actividad de inteligencia espacial-corporal: buscando un tesoro. Esta actividad resulta muy interesante ya que estimula además la lateralidad de los alumnos/as. Se trata de buscar tesoros escondidos en el patio del colegio y para conseguirlo tendrán que guiarse por un mapa del tesoro. Nuestros tesoros estarán escondidos en el patio del colegio. Para ello, se organizarán por equipos y cada equipo tiene destinado un tesoro que tendrá que buscar con ayuda del mapa que deberán de interpretar. Los tesoros serán nuevos materiales para el rincón científico. Esta actividad puede trabajarse con variantes empleando los distintos espacios del centro, y de esta forma podrá trabajarse una vez al trimestre. En nuestro programa de intervención 
neuropsicológica entendemos la evaluación como un proceso global, continuo y formativo que permita introducir elementos de mejora tanto en los procesos de enseñanza con en el aprendizaje. Por ello, dispondremos de una comisión evaluadora formada por el Equipo de Orientación Educativa y Psicopedagógica con frecuencia mensual y además dispondremos de momentos destinados a la información y colaboración de las familias o tutores legales de los alumnos por medio de circulares, reuniones generales y horarios de tutorías.

\section{Conclusiones}

Tras los resultados del análisis estadístico mediante texto, tablas y gráficos cabe conceder especialmente importancia a algunos aspectos objeto de nuestro estudio centrado en la mejora del proceso educativo en nuestros alumnos.

Comenzando por los datos obtenidos y representados acerca de las Inteligencias Múltiples es posible hacer una valoración las puntuaciones obtenidas de nuestra muestra alumnos/as. Tal y como viene reflejado en la figura 9 , si observamos la gráfica de las puntuaciones medias obtenidas del grupo-aula en cada una de las Inteligencias Múltiples podemos valorar que en inteligencia Lingüística presentan un desarrollo medio-alto, por otro lado en las inteligencias Espacial, Lógica-Matemática, CorporalKinestésica, Musical e Interpersonal se observan niveles de desarrollo medio y sin embargo, en las inteligencias Intrapersonal y Naturalista el alumnado presenta valoraciones medio-bajas siendo en esta última los niveles más bajos y deficitarios detectados. Si contemplamos los gráficos comprendidos de las figuras 1 a la 8 en la que aparecen las puntuaciones de los alumnos en cada una de las inteligencias resulta evidente que cada alumno/a presenta diferentes niveles de desarrollo deduciendo por tanto, que tratamos con un grupo de alumnos con unos ritmos de aprendizaje heterogéneos y con diferentes motivaciones y estilos cognitivos.

Prosiguiendo con nuestra descripción, si nos centramos en los datos aportados en lo que se refiere a aspectos de Creatividad resulta interesante detenerse en la figura 11, que presenta los porcentajes de valores que les asigna la tutora en niveles de creatividad al alumnado. Atendiendo a los ítems raramente y de vez en cuando en conductas de creatividad contemplamos a un $53 \%$ y $35 \%$ del total de conductas observadas en el grupo de alumnos/as, esto nos informaría que el $88 \%$ de conductas en alumnos/as de la muestra extraída presentan niveles de creatividad susceptibles de mejora y estimulación. No obstante no podemos obviar que un $12 \%$ del alumnado presentan un potencial aceptable creativo.

Aunque los resultados de esta investigación no presenten un rigor científico al respecto, podemos tratar de explicar la relación que pueda existir entre las Inteligencias y Creatividad. Para poder definir esta relación extraemos aquellos sujetos más potencialmente creativos de la muestra observados en la figura 10, corresponden así a los alumnos 22, 28 y 29. Si valoramos sus puntuaciones en Inteligencias Múltiples todas ellas presentan un desarrollo valorado en niveles medios y mediosaltos salvo en Inteligencia Naturalista como ocurre en el resto del alumnado. En principio y resumiendo lo expuesto, sí que podemos pensar que pueda existir una correlación entre niveles de Inteligencia y Creatividad en los sujetos. De todas formas, podemos partir también de la idea de que no todos los alumnos/as son creativos en todos las inteligencias propuestas tal y como expone Gardner (1983) al referirse a aspectos de creatividad en los sujetos y su relación con la inteligencia. Pueden existir alumnos con diferentes intereses y niveles de creatividad enfocados a diversas materias (pintura, danza, música, etc.) al igual que ocurre en las inteligencias. En este caso podría encontrarse el alumno 1. 
Finalizamos con el análisis del desarrollo de la Lateralidad de la muestra obtenida pudiendo interpretar un desarrollo lateral adecuado y definido en el $90 \%$ de los casos frente al $10 \%$ de desarrollo lateral menos definido observados en la figura 12. Estos datos no pueden ofrecer resultados definitivos y únicos pues debido a la edad de los sujetos es loable considerar que aún se encuentran en un momento evolutivo prestado al cambio y madurez junto con el asentamiento de la lateralidad hasta los 7 años de edad aproximadamente.

Si tratamos de indagar sobre la relación de la Creatividad con el asentamiento de la Lateralidad este estudio nos pone de evidencia que no existe correlación alguna, pues la mayor parte de los alumnos presentan aspectos de creatividad objeto de mejora y también en su mayoría el alumnado presenta un buen estado de lateralización.

\section{Referencias}

Amarís, M. (2002). Las múltiples inteligencias. Psicología desde el Caribe, 10, 27-38.

Antúnez, C. (2000). Estimular las inteligencias múltiples. Qué son. Cómo se manifiestan. Cómo funcionan. Madrid: Narcea.

Calero, M. (2011). Creatividad: reto de innovación educativa. México: Alfaomega Grupo Editor.

Ferrando, M., Prieto, MD., Ferrándiz, C. \& Sánchez, C. (2005). Inteligencia y Creatividad. Revista Electrónica de Investigación Psicoeducativa, 3(7), 21-50.

Gardner, H. (1995). Inteligencias múltiples. La teoría en la práctica. Barcelona: Paidós.

León, O. y Montero, I. (2004). Métodos de investigación en Psicología y Educación. Madrid: McGraw Hill.

Martín Lobo, MP. (2005). Lateralidad y rendimiento escolar. Madrid, Máster en Neuropsicología y Educación, Centro Universitario Villanueva.

Mayolas , MC., Villarroya, A. y Reverter, J. (2010). Relación entre la lateralidad y los aprendizajes escolares. Apuntes Educación Física y Deportes, 3(101), 32-42.

Ortiz, W. (2008). Neurociencia y Educación. Madrid: Alianza Editorial.

VV.AA. (1996). El desarrollo de la lateralidad infantil. Niños diestro- niño zurdo. Instituto Médico del Desarrollo Infantil. Barcelona: Lebon.

Pérez, L. y Beltrán, J. (2006). Dos décadas de inteligencias múltiples: implicaciones para la Psicología de la Educación. Papeles del Psicólogo, 27(3), 147-167.

Portellano, JA. (2008). Neuropsicología Infantil. Madrid: Síntesis.

Prieto, MD. y Ballester, P. (2003). Las inteligencias múltiples. Diferentes formas de enseñar y aprender. Madrid: Pirámide.

Rendón, M.A. (2009). Creatividad y cerebro: bases neuropsicológicas de la creatividad. Aula, 15, 117-135.

Sam Ed Brown. (1991). Experimentos de ciencias en educación infantil. Madrid: Narcea.

Sternberg, RJ. y O’Hara, L. (2005). Creatividad e inteligencia. Cuadernos de Información y comunicación, 10, 113-149.

\section{Webgrafía}

EI CSIC y FBBVA en la escuela. Aplicaciones: Ciencia en el aula. http://www.csicenlaescuela.csic.es/proyectos/proyectosdid.htm 